\title{
Análise de crescimento de girassol em Latossolo com diferentes níveis de saturação por bases no Cerrado(1)
}

\author{
Renato Fernando Amabile ${ }^{(2)}$, Daniel Pereira Guimarães ${ }^{(2)}$ e Austeclínio Lopes de Farias Neto(2)
}

Resumo - Com o objetivo de avaliar o comportamento de cinco cultivares de girassol em um Latossolo Vermelho argiloso, foram realizados quatro ensaios sob quatro níveis de saturação por bases (18\%, $35 \%, 56 \%$ e $77 \%$ ) obtidos com aplicação de calcário dolomítico. Os dados semanais de crescimento foram ajustados pelas funções logística, Weibull de três parâmetros e a logística generalizada de quatro parâmetros empregando-se o procedimento NLIN do SAS, bem como os processos interativos do método DUD. A função logística forneceu os melhores ajustes aos dados de crescimento. As cultivares foram categorizadas de acordo com o porte das plantas e tendências de crescimento a partir da análise de agrupamentos (cluster analysis), usando o método das ligações completas e a métrica da distância euclidiana. Todas as cultivares tiveram o crescimento reduzido quando o nível de saturação de bases foi de 18\%. A cultivar M734 apresentou bom desempenho no nível de 35\% de saturação por bases. Nos demais casos, os melhores resultados foram obtidos nos níveis de saturação por bases entre $56 \%$ e 77\%. As cultivares EMBRAPA 122 e M738 tiveram redução de crescimento quando a saturação foi elevada para 77\%. 'EMBRAPA 122' apresentou maior crescimento inicial e menor altura ao final do ciclo vegetativo.

Termos para indexação: Helianthus annuus, cultivar, calagem.

\section{Growth analysis of sunflower in a Cerrado Oxisol with different levels of basis saturation}

\begin{abstract}
In order to study the growth cycle of five sunflower cultivars, four experiments were carried out under four levels of basis saturation $(18 \%, 35 \%, 56 \%$ and $77 \%)$, obtained with the application of dolomitic limestone in an acidic Red Latosol, clay texture (Tipic Haplusthox). Weekly growth data were fitted using the Logistic, three parameters Weibull and four parameters Generalized Logistic (a special case of Richards function) functions. Among the models used, the Logistic function obtained was the best growth data fitting. The NLIN procedure of SAS statistical package was used for fitting the data through the interactive process of DUD method. Cultivars were grouped according to final size and growth rates by a cluster analysis using the complete linkage method and by the Euclidian distance. Sunflowers growth reduced in all cultivars at $18 \%$ basis saturation level. At $35 \%$ basis saturation level, the best results were achieved for cultivar M734. In other instances, the best growth results were obtained between 56\% and 77\%. For cultivars EMBRAPA 122 and M738, growth rates were reduced when basis saturation was increased to 77\%. The cultivar EMBRAPA 122 presented initial higher growth rate and lower plant height at the end of vegetative cycle.
\end{abstract}

Index terms: Helianthus annuus, cultivars, liming.

\section{Introdução}

O girassol (Helianthus annuus L.) responde por cerca de $13 \%$ de todo óleo vegetal produzido no mundo e apresenta um bom índice de crescimento

\footnotetext{
(1) Aceito para publicação em 24 de outubro de 2002.

(2) Embrapa-Centro de Pesquisa Agropecuária dos Cerrados, Caixa Postal 70.023, CEP 73301-970 Planaltina, DF. E-mail: amabile@cpac.embrapa.br, daniel@cpac.embrapa.br, auster@cpac.embrapa.br
}

em produção e área plantada (Estados Unidos, 2002). Por ser uma cultura de ampla adaptabilidade, alta tolerância à seca, alto rendimento de grãos e de óleo e pouco influenciada pela altitude e latitude, pode contribuir significativamente para maior diversificação dos sistemas agrícolas da região dos Cerrados, hoje restrita a poucas culturas. A expansão dessa cultura na região é favorecida por sua maior tolerância ao estresse hídrico (Castro et al., 1997), que ocorre normalmente no final do período chuvoso. 
Entretanto, é uma espécie sensível à acidez do solo, geralmente apresentando sintoma de toxidez de $\mathrm{Al}$ em pH em $\mathrm{CaCl}_{2}$ 0,1 $\mathrm{M}$ menor que 5,2 (Blamey et al., 1987; Wallace, 1990; Castiglioni, 1992), valor comum nos latossolos da região. No melhoramento genético dessa espécie para os Cerrados, uma importante característica a ser observada é a tolerância à acidez do solo, em especial, ao Al tóxico, presente na maioria dos solos dos Cerrados (Lopes \& Cox, 1977).

Em geral, os efeitos tóxicos do Al se manifestam inicialmente nas raízes (redução do crescimento, danos) e, com o agravamento do processo, no desenvolvimento de toda a planta. Como consequiência, as plantas apresentam redução de produtividade causada, entre outros fatores, pelo desequilíbrio nutricional e pelo estresse hídrico (Farias Neto et al., 1999).

Recentemente, no Brasil vêm sendo realizados trabalhos voltados ao desenvolvimento de métodos para seleção de genótipos tolerantes à acidez do solo (Astafeif, 1997; Farias Neto et al., 1999). A utilização desses genótipos e o uso de práticas de correção do solo constituem, portanto, alternativas para redução dos riscos de cultivo do girassol nos solos de Cerrado.

A resposta de cultivares de girassol à acidez do solo, pela análise de crescimento utilizada no pre- sente trabalho, já foi aplicada por Costa et al. (1984), Almeida \& Valle (1995) e Vieira et al. (1997) na avaliação de resposta de espécies ou cultivares a ambientes específicos.

O objetivo deste trabalho foi avaliar o comportamento de cinco cultivares de girassol sob quatro níveis de saturação por bases, em um Latossolo Vermelho argiloso.

\section{Material e Métodos}

Os quatro ensaios foram realizados em um Latossolo Vermelho argiloso, fase cerrado, álico, na Embrapa-Centro de Pesquisa Agropecuária dos Cerrados, Planaltina, DF. O clima, conforme classificação de Köppen, é do tipo Aw, denominado tropical de savana. A análise granulométrica $(0-20 \mathrm{~cm})$ do solo apresentou os seguintes resultados em $\mathrm{g} \mathrm{kg}^{-1}$ : areia grossa, 50; areia fina, 240; silte, 170 e argila, 540, determinados conforme Embrapa (1997). Aplicou-se calcário dolomítico com PRNT de $67,91 \%, 18,72 \%$ de $\mathrm{Ca}$ e $10,09 \%$ de $\mathrm{Mg}$, em $21 / 2 / 1997$. As doses de calcário foram calculadas conforme Sousa \& Lobato (1996), para elevar a saturação por bases, na camada de $0-20 \mathrm{~cm}$, a $20 \%, 35 \%, 50 \%$ e $70 \%$. O calcário foi incorporado com arado de discos. Os resultados das análises químicas do solo, antes e após a aplicação do calcário, encontram-se na Tabela 1.

A semeadura foi feita manualmente em 12/2/1998, em parcelas constituídas de quatro linhas de $5 \mathrm{~m}$. A área total

Tabela 1. Características químicas do solo na área dos ensaios, antes e após 12 meses da aplicação de calcário(1).

\begin{tabular}{|c|c|c|c|c|c|c|c|c|}
\hline $\begin{array}{l}\text { Profundidade } \\
\text { do solo }(\mathrm{cm})\end{array}$ & $\begin{array}{c}\text { pH em água } \\
(1: 2,5)\end{array}$ & $\begin{array}{c}\mathrm{P} \\
\left(\mathrm{mg} \mathrm{dm}^{-3}\right)\end{array}$ & $\mathrm{Ca}^{2+}$ & $\mathrm{Mg}^{2+}$ & $\begin{array}{c}\mathrm{K}^{+} \\
\mathrm{ol}_{\mathrm{c}} \mathrm{dm}\end{array}$ & $\mathrm{Al}^{3+}$ & $\mathrm{H}^{+}$ & $\begin{array}{l}\mathrm{V} \\
(\%)\end{array}$ \\
\hline & \multicolumn{8}{|c|}{ Antes da calagem } \\
\hline $0-20$ & 4,7 & 0,8 & 1,70 & 1,00 & 0,90 & 16,40 & 74,20 & 3,8 \\
\hline $20-40$ & 4,7 & 0,9 & 1,50 & 1,00 & 1,00 & 14,40 & 58,80 & 4,6 \\
\hline \multicolumn{9}{|c|}{ Após a calagem - $20 \%$ de saturação por bases } \\
\hline $0-20$ & 4,2 & 2,3 & 14,20 & 2,50 & 1,40 & 10,60 & 69,60 & 18 \\
\hline $20-40$ & 4,3 & 3,1 & 15,60 & 2,40 & 1,20 & 10,20 & 77,60 & 18 \\
\hline \multicolumn{9}{|c|}{ Após a calagem - 35\% de saturação por bases } \\
\hline $0-20$ & 4,7 & 3,2 & 30,87 & 5,03 & 1,90 & 2,70 & 67,10 & 35 \\
\hline $20-40$ & 4,6 & 1,5 & 20,81 & 3,39 & 2,00 & 5,70 & 64,70 & 27 \\
\hline \multicolumn{9}{|c|}{ Após a calagem - 50\% de saturação por bases } \\
\hline $0-20$ & 5,2 & 5,9 & 56,36 & 11,54 & 1,50 & 0,30 & 54,70 & 56 \\
\hline $20-40$ & 4,5 & 0,9 & 12,20 & 2,50 & 1,20 & 10,50 & 62,70 & 18 \\
\hline \multicolumn{9}{|c|}{ Após a calagem - 75\% de saturação por bases } \\
\hline $0-20$ & 5,6 & 4,2 & 63,88 & 14,02 & 1,10 & 0,00 & 23,80 & 77 \\
\hline $20-40$ & 5,3 & 1,1 & 30,67 & 6,73 & 0,80 & 1,20 & 44,80 & 45 \\
\hline
\end{tabular}

${ }^{(1)}$ As quantidades de calcário aplicadas foram calculadas para que a saturação de bases do solo atingissem os níveis de $20 \%, 35 \%, 50 \%$ e $75 \%$. 
da parcela era de $16,0 \mathrm{~m}^{2}$ e a área útil, de $6,4 \mathrm{~m}^{2}$. O preparo do solo constou da incorporação dos restos culturais de soja, com arado de discos, seguido de grade niveladora. Aplicou-se o herbicida Trifluralin na dosagem de 1,8 $\mathrm{L} \mathrm{ha}^{-1}$. Em seguida, os sulcos de semeadura foram abertos mecanicamente e neles aplicados $18 \mathrm{~kg} \mathrm{ha}^{-1} \mathrm{de} \mathrm{N}$ (uréia), $135 \mathrm{~kg} \mathrm{ha}^{-1}$ de $\mathrm{P}_{2} \mathrm{O}_{5}$ (superfosfato simples) e $72 \mathrm{~kg} \mathrm{ha}^{-1}$ de $\mathrm{K}_{2} \mathrm{O}$ (cloreto de potássio) e 1,0 $\mathrm{kg} \mathrm{ha}^{-1}$ de B (bórax). Aos 30 dias após a emergência, aplicaram-se, em cobertura, $90 \mathrm{~kg} \mathrm{ha}^{-1}$ de $\mathrm{N}$ (uréia). A população das cinco cultivares foi de 42.000 plantas ha $^{-1}$. O delineamento experimental foi o de blocos ao acaso, com cinco tratamentos (cultivares EMBRAPA 122, DK180, C11, M734 e M738) e quatro repetições.

A altura das plantas foi medida aos 12 dias após a emergência e, semanalmente, até a maturação fisiológica. A tendência do crescimento foi avaliada pelo ajuste de funções não lineares assintóticas. A seleção dos modelos baseou-se em uma análise prévia das tendências do crescimento das cultivares a partir de plotagem gráfica, optando-se, desta forma, pela seleção de modelos capazes de descrever distribuições simétricas.

Foram empregadas as funções logística, logística generalizada de quatro parâmetros (Nelder, 1961), a qual constitui caso particular da função Richards (Richards, 1959), e a função Weibull de três parâmetros (Weibull, 1951), com as seguintes configurações:

Logística: $\omega=\theta /\left(1+\exp ^{\alpha-\beta * X}\right)$

Logística generalizada: $\omega=\theta /\left(1+\exp ^{\alpha-\beta * X}\right)^{\gamma}$

Weibull: $\omega=\theta\left(1-\exp ^{-\beta * X^{\gamma}}\right)$.

Nos modelos, $\omega$ expressa a magnitude da variável dependente (altura das plantas); $\theta$ é o parâmetro assintótico (correspondendo à altura na maturação); $\alpha$ corresponde ao parâmetro de locação; $\beta$ determina a taxa de expansão do crescimento; $\gamma$ define o ponto de inflexão; exp referese à base dos logaritmos neperianos e $\mathrm{X}$ refere-se ao tempo em que o crescimento ocorre. Mais detalhes sobre as funções logística e logística generalizada são encontrados em Johnson et al. (1995) e sobre a função Weibull, em Evans et al. (2000). O ajuste das funções foi efetuado aplicando-se o procedimento NLIN do Programa SAS (SAS Institute, 1999). A seleção do modelo mais adequado para explicar o crescimento das plantas teve como base a precisão do ajuste e a simplicidade das funções, definidas na seguinte ordem: logística, Weibull e logística generalizada. A comparação entre os modelos, em razão da precisão dos ajustamentos, obedeceu os seguintes critérios: função logística versus logística generalizada e função logística versus função Weibull. No primeiro caso, tem-se a comparação do modelo completo (logística generalizada) com um modelo reduzido (logística), em que o parâmetro $\gamma$ é suprimido. Para tal, utilizou-se a estatística F dada por:

$$
\mathrm{F}=\frac{(\mathrm{SQRr}-\mathrm{SQRc}) /(\mathrm{GLr}-\mathrm{GLc})}{\mathrm{SQRc} / \mathrm{GLc}}
$$

em que SQRr refere-se à soma de quadrados do modelo reduzido (logística); SQRc é a soma de quadrados do modelo completo (logística generalizada) e GLr e GLc são os graus de liberdade do resíduo relativos aos respectivos modelos. O nível de significância de F calculado é comparado com os valores tabelados com GLr-GLc graus de liberdade no numerador e GLc graus de liberdade no denominador. No caso da função logística versus função Weibull ou logística generalizada versus função Weibull, a comparação se deu por meio do coeficiente de determinação $\left(\mathrm{R}^{2}\right)$ para modelos não-lineares, cuja fórmula é: $\mathrm{R}^{2}=1$ - SQR/SQTc,

em que $\mathrm{SQR}$ refere-se à soma de quadrados dos resíduos e SQTc à soma de quadrados totais.

A classificação das cultivares em relação ao hábito de crescimento e porte final das plantas foi feita mediante análise de agrupamentos (cluster), usando a métrica da distância euclidiana e o método de ligações completas envolvendo as posições referentes aos valores assintóticos, e a idade em que ocorre o ponto de inflexão da curva de crescimento ajustada pelo modelo não-linear. Assim, dois tratamentos seriam considerados semelhantes se as cultivares apresentassem altura das plantas e hábitos de crescimentos similares.

\section{Resultados e Discussão}

A função logística, embora seja o modelo mais simples, apresentou resultados similares à função logística generalizada, exceto em relação à cultivar DK180 a $18 \%$ de saturação por bases, indicando que a inclusão do parâmetro $\gamma$ no modelo apresentou baixa contribuição para a precisão dos ajustes (Tabela 2). Também a função Weibull, cuja flexibilidade permite descrever famílias de curvas sigmoidais com diferentes tendências, não apresentou resultados superiores aos obtidos pela função logística, conforme os valores dos coeficientes de determinação $\left(\mathrm{R}^{2}\right)$.

Esses resultados indicam que as taxas de crescimento das cultivares testadas tenderam a mostrar distribuições simétricas cuja configuração é semelhante à distribuição normal, independentemente do tratamento adotado. Tais resultados são contrastantes com os observados na maioria das espécies vegetais 
cujas tendências de crescimento apresentam assimetria positiva, o que pode ser constatado pela larga utilização das funções de Gompertz e Richards. Christoffoleti (2001) aplicou a função Richards para descrever o crescimento de picão-preto e Bedmar et al. (1999) usaram a função Gompertz para avaliar os danos da infestação de plantas daninhas sobre a cultura do milho. A maior demora do girassol em atingir o ponto de inflexão indicou que essa cultura requer mais tempo para promover a cobertura do solo. A cultivar DK180 atingiu apenas a metade de sua altura final aos 40 dias após a germinação (Figura 1). Assim, a adoção de práticas culturais, como o plantio direto, deveria ser considerada para a instalação das lavouras de girassol.

Os resultados do ajuste da função logística evidenciaram o efeito dos níveis de saturação por bases sobre a altura das plantas (valor assintótico) e as diferentes tendências de crescimento das cultivares (ponto de inflexão) (Tabela 3).
As diferentes tendências, relacionando os tratamentos mais antagônicos, ou seja, a cultivar EMBRAPA 122 crescendo em um nível de saturação por bases de 35\%, em que o ponto de máxima expansão do crescimento ocorre a cerca de 30 dias após a emergência, e a cultivar DK180, no nível de saturação por bases de $18 \%$, no qual esse ponto ocorre em torno de 40 dias, são mostradas na Figura 1.

A taxa de expansão do crescimento do girassol foi inversamente proporcional ao nível de correção da acidez do solo. Isto significa que o plantio dessa espécie em solos com baixos níveis de saturação por bases resulta em redução do crescimento das plantas e, conseqüentemente, redução da cobertura do solo.

A classificação das funções por meio da análise de "cluster" evidencia a formação de três grupos distintos, determinados pela altura das plantas, e os subgrupos definidos pela combinação do porte e das taxas de expansão do crescimento (precocidade) (Figura 2). Todos os tratamentos referentes ao nível

Tabela 2. Precisão das estimativas, expressas pela estatística $\mathrm{F}$ e pelo coeficiente determinístico $\left(\mathrm{R}^{2}\right)$ para as funções logística (L), logística generalizada (LG) e Weibull (W) ajustadas aos dados de crescimento em altura das cultivares de girassol em quatro níveis de saturação por bases do solo ${ }^{(1)}$.

\begin{tabular}{|c|c|c|c|c|}
\hline \multirow[t]{2}{*}{ Cultivar } & \multirow{2}{*}{$\begin{array}{c}\text { Estatística F } \\
\text { L e LG }\end{array}$} & \multicolumn{3}{|c|}{ Coeficiente determinístico $\left(\mathrm{R}^{2}\right)$} \\
\hline & & $\mathrm{L}$ & LG & $\mathrm{W}$ \\
\hline & \multicolumn{4}{|c|}{ Saturação por bases de $18 \%$} \\
\hline C11 & $1,95^{\mathrm{ns}}$ & 0,9868 & 0,9876 & 0,9857 \\
\hline DK180 & $10,39^{* *}$ & 0,9886 & 0,9914 & 0,9869 \\
\hline M734 & $2,08^{\mathrm{ns}}$ & 0,9894 & 0,9900 & 0,9883 \\
\hline M738 & $1,97^{\mathrm{ns}}$ & 0,9887 & 0,9989 & 0,9879 \\
\hline \multirow[t]{2}{*}{ EMBRAPA 122} & $0,30^{\mathrm{ns}}$ & 0,9658 & 0,9661 & 0,9645 \\
\hline & \multicolumn{4}{|c|}{ Saturação por bases de $35 \%$} \\
\hline C11 & $0,74^{\mathrm{ns}}$ & 0,9911 & 0,9913 & 0,9907 \\
\hline DK180 & $1,59^{\mathrm{ns}}$ & 0,9929 & 0,9933 & 0,9926 \\
\hline M734 & $0,07^{\mathrm{ns}}$ & 0,9915 & 0,9916 & 0,9916 \\
\hline M738 & $0,05^{\mathrm{ns}}$ & 0,9927 & 0,9927 & 0,9927 \\
\hline \multirow[t]{2}{*}{ EMBRAPA 122} & $0,03^{\mathrm{ns}}$ & 0,9637 & 0,9638 & 0,9626 \\
\hline & \multicolumn{4}{|c|}{ Saturação por bases de $56 \%$} \\
\hline C11 & $1,27^{\mathrm{ns}}$ & 0,9944 & 0,9946 & 0,9939 \\
\hline DK180 & $1,00^{\mathrm{ns}}$ & 0,9901 & 0,9904 & 0,9900 \\
\hline M734 & $0,08^{\mathrm{ns}}$ & 0,9962 & 0,9962 & 0,9963 \\
\hline M738 & $0,28^{\mathrm{ns}}$ & 0,9935 & 0,9935 & 0,9935 \\
\hline \multirow[t]{2}{*}{ EMBRAPA 122} & $0,26^{\mathrm{ns}}$ & 0,9871 & 0,9872 & 0,9863 \\
\hline & \multicolumn{4}{|c|}{ Saturação por bases de $77 \%$} \\
\hline C11 & $0,29^{\mathrm{ns}}$ & 0,9947 & 0,9948 & 0,9945 \\
\hline DK180 & $1,09^{\mathrm{ns}}$ & 0,9953 & 0,9955 & 0,9955 \\
\hline M734 & $0,00^{\mathrm{ns}}$ & 0,9919 & 0,9919 & 0,9920 \\
\hline M738 & $0,03^{\mathrm{ns}}$ & 0,9810 & 0,9810 & 0,9810 \\
\hline EMBRAPA 122 & $0,02^{\mathrm{ns}}$ & 0,9913 & 0,9913 & 0,9907 \\
\hline
\end{tabular}




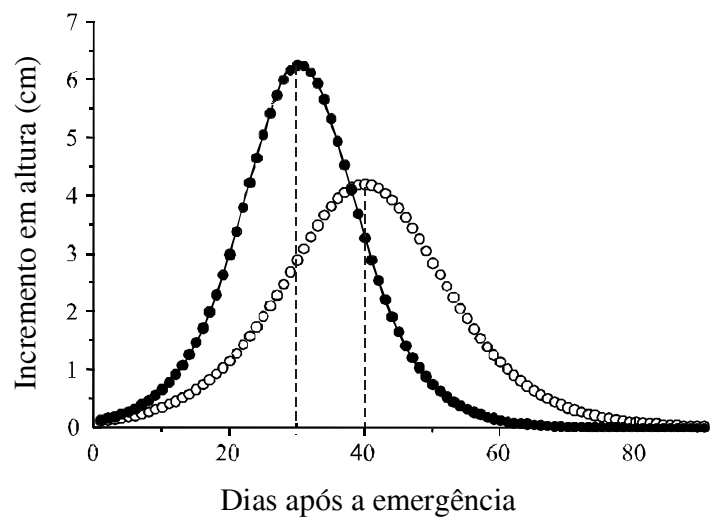

Figura 1. Crescimento de plantas de girassol, cultivares EMBRAPA 122 (•) e DK180 (o), sob 35\% e 18\% de saturação por base do solo, respectivamente.

Tabela 3. Parâmetros estimados, de locação (B0) e de forma (B1), para ajuste da função logística e pontos de inflexão do crescimento de plantas de girassol em razão da idade para os diferentes níveis de saturação por bases do solo (V).

\begin{tabular}{ccccc}
\hline $\mathrm{V}^{(1)}(\%)$ & Assíntota & B0 & B1 & Inflexão \\
\hline \multicolumn{5}{c}{ C11 } \\
18 & 145,036 & 4,76792 & 0,12481 & 38,20 \\
35 & 163,106 & 4,75375 & 0,13000 & 36,57 \\
56 & 167,341 & 4,69156 & 0,12897 & 36,38 \\
77 & 172,764 & 5,07625 & 0,14065 & 36,09 \\
\hline \multicolumn{5}{c}{ DK180 } \\
18 & 132,361 & 5,00050 & 0,12697 & 39,38 \\
35 & 159,867 & 5,04192 & 0,13865 & 36,36 \\
56 & 171,488 & 4,98544 & 0,13790 & 36,15 \\
77 & 170,976 & 5,14619 & 0,14246 & 36,12 \\
\hline \multicolumn{5}{c}{ M734 } \\
18 & 141,104 & 4,55668 & 0,12294 & 37,06 \\
35 & 167,525 & 4,69733 & 0,13279 & 35,37 \\
56 & 165,324 & 4,44540 & 0,13262 & 33,52 \\
77 & 169,370 & 4,68751 & 0,13810 & 33,94 \\
\hline \multicolumn{5}{c}{ M738 } \\
18 & 144,654 & 4,60335 & 0,12445 & 36,99 \\
35 & 155,849 & 4,61599 & 0,13346 & 34,59 \\
56 & 168,003 & 4,48514 & 0,13386 & 33,51 \\
77 & 161,701 & 4,83675 & 0,14830 & 32,61 \\
\hline \multicolumn{5}{c}{ EMBRAPA 122 } \\
18 & 132,783 & 5,27566 & 0,17136 & 30,79 \\
35 & 142,344 & 5,25806 & 0,17608 & 29,86 \\
56 & 160,481 & 4,74053 & 0,15547 & 30,49 \\
77 & 151,659 & 5,09950 & 0,16886 & 30,20 \\
\hline (1)Valores de saturação por bases medida após 12 meses da aplicação do \\
calcário.
\end{tabular}

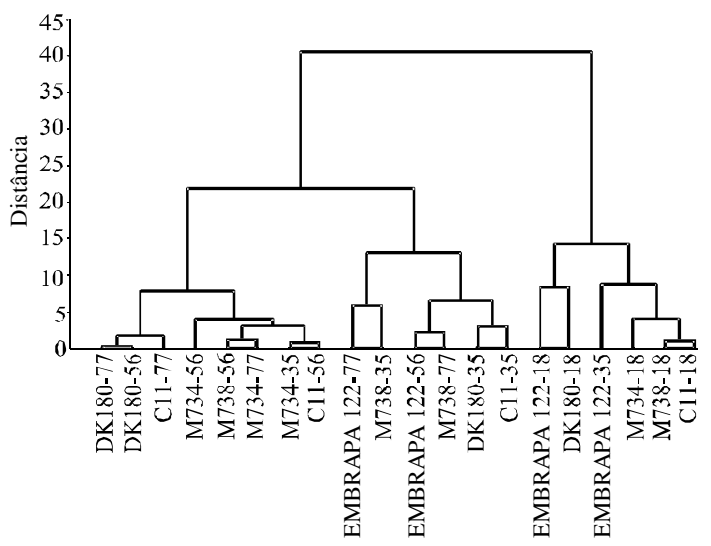

Figura 2. Classificação hierárquica das curvas de crescimento de cultivares de girassol usando o método de ligações completas e a métrica da distância euclidiana.

de saturação por bases de $18 \%$ estão agrupados no grupo de menor porte, indicando que as cultivares apresentam redução de crescimento nessas condições de solo.

A cultivar EMBRAPA 122 apresentou menor altura e maior crescimento inicial em relação às demais, o que sugere a possibilidade de seu cultivo em menores espaçamentos, contribuindo, com isso, no controle de plantas daninhas. A cultivar C11 respondeu positivamente ao aumento dos níveis de saturação por bases. A cultivar DK180 não expressou resposta em razão da elevação do nível de saturação por bases de $56 \%$ para $77 \%$. No caso da cultivar M 734, os resultados foram similares em relação aos níveis de saturação por bases de 35\%, 56\% e 77\%, o que a torna recomendável para solos mais ácidos. As cultivares EMBRAPA 122 e M 738 tiveram crescimento reduzido quando o nível de saturação por bases passou de $56 \%$ para $77 \%$. Esses resultados sugerem a necessidade de seleção do material genético bem como a introdução de genótipos de girassol no sistema de produção dos Cerrados em razão da fertilidade dos solos.

\section{Conclusões}

1. Existe relação inversa entre o nível de saturação por bases e o ponto de inflexão da curva de crescimento do girassol. 
2. O crescimento das plantas é reduzido quando o nível de saturação por bases do solo situa-se em $18 \%$.

3. A cultivar EMBRAPA 122 apresenta maior crescimento inicial e menor porte de planta ao final do ciclo vegetativo, o que possibilita o aumento do número de plantas por unidade de área e redução nos tratos culturais.

4. O crescimento da cultivar M734 é semelhante nos níveis de $35 \%$, $56 \%$ e $77 \%$ de saturação por bases, o que demonstra sua melhor adaptação em solos menos corrigidos.

5. As cultivares M738 e EMBRAPA 122 manifestam redução no crescimento quando o nível de saturação por bases é aumentado de $56 \%$ para $77 \%$.

6. A seleção da cultivar de girassol a ser plantada é influenciada pelas condições de saturação por bases do solo.

\section{Referências}

ALMEIDA, A. A. F. de; VALLE, R. R. Análise de crescimento do fruto e das sementes de sete genótipos de Theobroma cacao L. Pesquisa Agropecuária Brasileira, Brasília, v. 30, n. 7, p. 909-916, jul. 1995.

ASTAFEIF, N. C. Identificação em hidroponia de genótipos de girassol (Helianthus annuus, L.) tolerantes ao alumínio. 1997. 99 f. Dissertação (Mestrado em Genética e Melhoramento) - Universidade Estadual de Londrina, Londrina, 1997.

BEDMAR, F.; MANETTI, P.; MONTERUBBIANESI, G Determination of the critical period of weed control in corn using a thermal basis. Pesquisa Agropecuária Brasileira, Brasília, v. 34, n. 2, p. 187-193, fev. 1999.

BLAMEY, F. P. C.; EDWARDS, D. G.; ASHER, C. J. Nutritional disorders of sunflower. Brisbane: University of Queensland, 1987. $72 \mathrm{p}$.

CASTIGLIONI, V. B. R. Cultivo do girassol. Londrina: Embrapa-CNPSo, 1992. 4 p. (Circular Técnica, 13).

CASTRO, C. de; CASTIGLIONI, V. B. R.; BALLA, A.; LEITE, R. M. V. B. de C.; KARAM, D.; MELLO, H. C.; GUEDES, L. C. A.; FARIAS, J. R. B. A cultura do girassol. Londrina: Embrapa-CNPSo, 1997. 36 p. (Circular Técnica, 13).

CHRISTOFFOLETI, P. J. Análise comparativa do crescimento de biótipos de picão-preto (Bidens pilosa) resistente e suscetível aos herbicidas inibidores da ALS. Planta Daninha, Curitiba, v. 19, n. 1, p. 75-83, 2001.
COSTA, C.; HAAG, H. P.; PITELLI, R. A. Análise de crescimento da Galactia striata (Jacq.) Urb., em função de sete épocas de semeadura em um Latossolo VermelhoEscuro álico, na região de Ilha Solteira, SP. Anais da Escola Superior de Agricultura Luiz de Queiroz, Piracicaba, v. 41, n. 1, p. 383-412, 1984.

EMBRAPA. Centro Nacional de Pesquisa de Solos (Rio de Janeiro, RJ). Manual de métodos de análise de solo. 2. ed. rev. atual. Rio de Janeiro, 1997. 212 p.

ESTADOS UNIDOS. Foreign Agricultural Service. Oilseeds: world markets and trade. Table 1. (Circular Series FOP 08-02). Disponível em: <http://www.fas.usda.gov/oilseeds/ circular/2002/02-08/toc.htm>. Acesso em: 28 ago. 2002.

EVANS, M.; HASTINGS, N.; PEACOCK, B. Statistical distributions. 3rd ed. New York: J. Wiley, 2000. 221 p.

FARIAS NETO, A. L.; ANDRADE, L. R. M. de; OLIVEIRA, M. F. Metodologia para seleção de genótipos de girassol (Helianthus annuus L.) tolerantes ao alumínio. Planaltina: Embrapa-CPAC, 1999. 2 p. (Pesquisa em Andamento, 25).

JOHNSON, N. L.; KOTZ, S.; BALAKRISHNAN, N. Continuous univariate distributions. 2nd ed. New York: J. Wiley, 1995. v. 2.

LOPES, A. S.; COX, F. R. A survey of the fertility status of surface soils under "Cerrado" vegetation in Brazil. Soil Science Society of America Journal, Madison, v. 41, n. 4, p. 742-747, 1977.

NELDER, J. A. The fitting of a generalization of the logistic curve. Biometrics, Washington, v. 17, n. 1, p. 89110, 1961.

RICHARDS, F. J. A flexible growth function for empirical use. Journal of Experimental Botany, Oxford, v. 10, n. 29, p. 290-300, 1959.

SAS INSTITUTE (Cary, Estados Unidos). SAS/STAT user's guide: NLIN procedure: version 8. Cary, 1999. v. 1.

SOUSA, D. M. G. de; LOBATO, E. Correção do solo e adubação da soja. Planaltina: Embrapa-CPAC, 1996. 30 p. (Circular Técnica, 33).

VIEIRA, D. J.; BELTRÃO, N. E. de M.; AZEVEDO, D. M. P. de; NÓBREGA, L. N. da; COSTA, L. B. da; ARAÚJO, J. D. de. Análise de crescimento do gergelim nos Cariris Velhos da Paraíba: efeito de cultivares e de configurações de plantio. Campina Grande: EmbrapaCNPA, 1997. 4 p. (Comunicado Técnico, 56).

WALLACE, A. Interactions of water stress and aluminum stress in barley and sunflower cultivars. Journal of Plant Nutrition, New York, v. 13, n. 3/4, p. 425-436, 1990.

WEIBULL, W. A statistical distribution function of wide applicability. Journal of Applied Mechanics, Fairfield, v. 18 , n. 3 , p. $293-297,1951$. 